\title{
Kinetics Analysis of Pelvis, Thorax, and Bowling Arm in Cricket Bowling
}

\author{
Rene Ferdinands
}

\begin{abstract}
In this study, a three-dimensional (3D) dynamics model of the human body was developed to analyze the motion of fast bowling in cricket. Nine fast bowlers (22.4 \pm 3.2 years) were selected from high-level regional cricket to bowl a series of balls at a target placed approximately on a 'good length' in line with the wickets, while their bowling actions were captured by a 10-camera $240 \mathrm{~Hz}$ motion analysis system (Motion Analysis Corp.). Motion analysis data were obtained from the tracked markers on the bowler strategically placed on the body to define a 3D joint coordinate system for each segment. Two Bertec force plates were used to measure the ground reaction forces. The resulting kinematic and force plate data of the fastest ball were fed into a computer model designed using the Mechanical Systems Pack (Wolfram Research, Inc., V. 5.2), a set of Mathematica packages written for the analysis of spatial rigid body mechanisms, implementing a dynamics formulation with Lagrangian multipliers. The computer model gave a 3D representation of the human body as a system of 15 rigid body segments with mass and inertia properties. Inverse solution dynamics were calculated to generalize certain fundamental aspects of the pelvis, thorax and bowling arm during fast bowling.
\end{abstract}

Keywords: Biomechanics, Cricket, Fast bowling, Kinetics.

How to cite this article: Ferdinands R. Kinetics Analysis of Pelvis, Thorax, and Bowling Arm in Cricket Bowling. J Postgrad Med Edu Res 2015;49(4):159-163.

Source of support: Nil

Conflict of interest: None

\section{INTRODUCTION}

The function of bowling in cricket is to deliver the ball to the batter such that the ball first bounces once off the ground, the action constrained by the laws of cricket, specifying that any straightening of the bowling arm must occur well before the time of ball release. ${ }^{1}$ Formal research in the biomechanics of bowling has been focused on two areas: the identification of factors that increase a bowler's susceptibility to injury, particularly

Lecturer

Faculty of Health Sciences, University of Sydney, Sydney Australia

Corresponding Author: Rene Ferdinands, Lecturer, Faculty of Health Sciences, University of Sydney, Sydney, Australia, Phone: 61293519776, e-mail: edouard.Ferdinands@sydney.edu.au of the lower lumbar region; and the correlation of kinematic variables and sequences with elite fast bowlers. Such research has identified the mixed action as a potential cause of back injury, and found correlations between certain kinematic characteristics and ball release speed. ${ }^{2}$ Some research has also made a preliminary analysis of segmental sequencing, based on peak temporal data of kinematic variables. However, kinematics data is limited, able to specify the critical positional features of bowling technique; in many cases also generate an accurate descriptive account of bowling technique, but unable to establish the causal mechanisms underlying the motion sequence of bowling.

Since it is a maxim of physics that an external torque applied about the pivot of a lever will cause that lever to angularly accelerate, the human body is effectively a coupled system of musculoskeletal levers, requiring a coordinated generation of joint torque inputs to achieve a desired movement sequence. Hence, to bowl a ball, bowlers have to generate an appropriate set of joint torque histories to coordinate the movements of limbs in order to satisfy the constraints of the bowling action. The selection of joint torque histories ultimately determines whether the bowling action has achieved its purpose, which in fast bowling is to control the kinematics of the bowling hand, so that the ball is delivered with high velocity and precision. Bowling is, therefore, a highly coordinated task, particularly since the bowling action must be combined with a run-up phase to generate linear momentum. An analysis of bowling technique, therefore, is not amenable to simple kinematic analysis. Comprising so many different mechanical elements, a technical analysis of bowling requires an integrated analysis of kinetics, something that requires the simulation of dynamics models with fast motion analysis data.

In this study, a rigid body dynamics model of the human body was designed using the Newton-Euler dynamics formulation with Lagrangian multipliers. The aim was to investigate the fundamental kinetics properties of bowling actions in a sample of elite fast bowlers; thus, providing information on the actuating joint torques of the major body segments in fast bowling. 


\section{MATERIALS AND METHODS}

Nine elite male fast bowlers, who all delivered the ball in excess of $125 \mathrm{~km} / \mathrm{h}$, were selected for the study. We used a 10-camera $240 \mathrm{~Hz}$ Eva motion analysis system (Motion Analysis Corp.) to track 45 markers placed on the limbs of each bowler, while bowling a series of balls at maximum effort. Each bowler had to bowl six balls on an artificial surface at a target (the stumps) 20 m away, at the same time ensuring to make rear foot and front foot contacts on two Bertec force plates $(960 \mathrm{~Hz})$ during the delivery stride. Hence, the capture volume encompassed the positions of back foot contact, front foot contact, and ball release. The Eva system was calibrated according to the manufacturer's recommendations, achieving a precision of less than $1 \mathrm{~mm}$ residual error in marker position. The ball with the fastest ball release speed was selected for analysis.

All marker positions were subject to the general condition that they had to define a three-dimensional orthogonal joint coordinate system for their respective segments. ${ }^{3}$ The orientations of each joint coordinate system were calculated in Mathematica (Wolfram Research Ltd., V. 5.2) as z-y-x Euler angle sequences. A total of 15 joint coordinate systems were defined, representing each of the major body segments: head and neck (as one segment), thorax, lower trunk, thighs, shanks, feet, upper arms, forearms, and hands. Each of these segments were assigned the full set of mass-inertia properties (mass, centre of mass and moment of inertia) in a program written in the Mechanical Systems Pack, a set of Mathematica (Version 5.2) packages designed to assist in the analysis and design of spatial rigid body mechanisms. ${ }^{4}$

Since each rigid body in 3D space has 6 degree of freedom (Dof), 15 segments moving independently have $90^{\circ}$ Dof (i.e. 6 Dof $\times 15$ segments). However, to incorporate a system of multiple rigid bodies in a dynamic model of bowling, these rigid bodies must form a mechanism resembling the functional movement of joints in the human body, something that was achieved with spherical joint constraints. The advantage of the spherical joint constraint is that it constrains three Dof, linking segments in a way that is mechanically equivalent to that of the human ball-and-socket joint, allowing flexion-extension, adduction-abduction, and internal-external rotation-the complete range of anatomical movements. By writing a program in the Mechanical Systems Pack to link the 15 rigid body segments using spherical joints, the total Dof of the dynamic model was reduced 45 ; but to account for the translation of the whole body, a relative distance driving constraint was applied to a virtual joint center defined at the mid-hips. In addition, for the shoulder joints, two relative distance driving constraints were used, required to make each shoulder joint movable relative to the thorax segment. Since linear driving constraints add three Dof to a mechanical system, the addition of these three driving constraints, increased the Dof of the model by nine, making the total Dof of the 15 segment rigid body model now equal to 54 .

With all the segment linkages in place, the model was simulated in the Mechanical Systems Pack by using angular driving constraints, specified in terms of the $z-y-x$ Euler angle sequences calculated at each time point during the bowling action. These Euler angle sequences oriented each joint coordinate system with respect to the global coordinate system by concatenating the joint-link transformation matrices throughout each kinematic chain. Then, the segments were dynamically linked together using holonomic constraint equations. External loads to the segments, such as gravitational forces, ground reaction forces, and ground reaction moments were determined from force plate data. These external loads were applied to the model by means of time-switching constraints, effectively giving the model a load switching capability explicitly dependent on time. The Mechanical Systems Pack generated the dynamics equations of motion using a Newtonian-Lagrange Multiplier method. The equations were then solved iteratively using the Newton-Raphson method. ${ }^{5}$ Finally, the Lagrange Multipliers were used post-solution to calculate the reaction loads applied by each constraint to its respective body segment, thereby, calculating the external joint torques and forces (kinetics) on the body during the bowling action.

The kinetics data were calculated over the arm acceleration phase, when the bowling arm undergoes its highest period of angular acceleration, a phase approximately corresponding to the time interval from front foot contact to ball release.

\section{RESULTS}

The mean pelvis joint torques calculated during the bowling arm acceleration phase were pelvis flexion $(-136.7 \pm 54.3 \mathrm{~N} \mathrm{~m})$, pelvis rotation $(-138.9 \pm 62.5 \mathrm{~N} \mathrm{~m})$, and pelvis tilt $(-115.6 \pm 68.9 \mathrm{~N} \mathrm{~m})$. The time history of pelvis rotation and pelvis rotation torques were similar, their peaks occurring at 25 and 35\%, respectively; both torques generally acting to the left. However, the pelvis flexion-extension torque was notably different from these torques. It was an increasing function with no discernible peaks, always operating in the direction of extension, its highest value occurring at ball release (Fig. 1). Normalized with respect to mass multiplied length, the peak 
values of pelvis flexion, pelvis rotation and pelvis tilt were $-1.2 \mathrm{~N} \mathrm{~kg}^{-1},-1.6 \mathrm{~N} \mathrm{~kg}^{-1}$ and $-1.5 \mathrm{~N} \mathrm{~kg}^{-1}$, respectively.

The mean thorax joint torques calculated during the bowling arm acceleration phase were pelvis flexion (45.2 \pm $51.9 \mathrm{~N} \mathrm{~m})$, thorax rotation $(62.2 \pm 26.2 \mathrm{~N} \mathrm{~m})$, and thorax tilt $(57.3 \pm 67.6 \mathrm{~N} \mathrm{~m})$. The time histories of thorax torques were noticeably different from the pelvis torques in terms of their peak values, direction and general patterning (Fig. 2). The thorax flexion-extension torque rotation acted in the direction of flexion from 0 to $80 \%$ of the phase, reaching a peak value at $22 \%$, before acting in the direction of extension from $81 \%$ to ball release. The thorax rotation torque always operated in the direction of left rotation, achieving its highest values from 0 to $30 \%$, during which there was a peak plateau period from 0 to $18 \%$ (although the highest value was marginally highest at $0 \%$ ). Thorax lateral bending torque was always to the left, reaching a peak relatively later in the phase at $92 \%$, and maintaining a high value through to ball release. Normalized with respect to mass multiplied length, the peak values of thorax flexion-extension, thorax rotation and thorax lateral bending were $0.62 \mathrm{~N} \mathrm{~kg}^{-1}, 0.66 \mathrm{~N} \mathrm{~kg}^{-1}$ and $0.76 \mathrm{~N} \mathrm{~kg}^{-1}$, respectively.

The bowling arm torques were generally of a lower magnitude than either the pelvis or thorax torques, and their peak values generally occurred later in the arm acceleration phase (Fig. 3). The shoulder flexion-extension torque acted in the direction of flexion from 0 to $60 \%$, gradually decreasing during this period, and reversing at $61 \%$, acting in the direction of extension until reaching a peak value at ball release. The shoulder adductionabduction torque, on the other hand, always acted in the direction of adduction, increasing its value until $93 \%$, when it reached a peak, and only slightly dropping below this peak value at ball release. The elbow

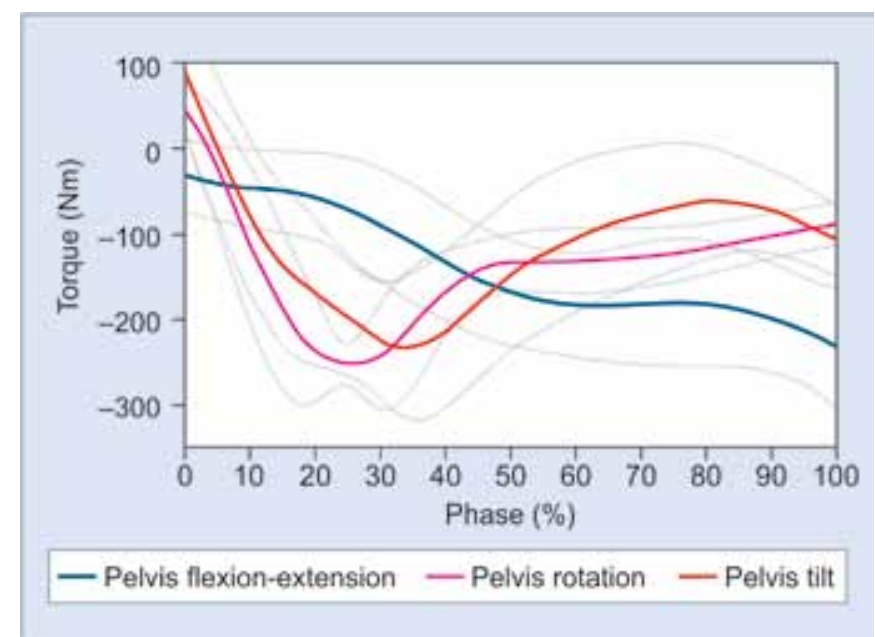

Fig. 1: Ensemble average (mean $\pm S D$ ) of time-varying joint torques of pelvis flexion, pelvic rotation and pelvis tilt. Flexion, left rotation and left tilt were defined as positive flexion-extension torque always acted in the direction of flexion, smaller than the values of the shoulder torques, reaching a peak at $43 \%$, and then decreasing until ball release. The wrist flexion torque was the smallest of the torques, but rapidly increased in value from $78 \%$, reaching a peak value at $91 \%$, close to ball release. Normalized with respect to mass multiplied length, the peak torque values of shoulder extension, shoulder adduction, elbow flexion, and wrist flexion were $0.64 \mathrm{~N} \mathrm{~kg}^{-1}, 0.67 \mathrm{~N} \mathrm{~kg}^{-1}, 0.20 \mathrm{~N} \mathrm{~kg}^{-1}$ and $0.021 \mathrm{~N} \mathrm{~kg}^{-1}$, respectively.

A comparative analysis of the kinetics sequencing in terms of the temporal order of peak joint torques shows that pelvis rotation and pelvis tilt were actuated early in the phase, generating noticeable peak values at $25 \%$ and $35 \%$, respectively. However, the thorax rotation torque may be considered to have acted first, having no discernible peak, but achieving its highest values in the period from 0 to $18 \%$ during early front foot contact (Fig. 2). All the other torques achieved their maximum values relatively later during the arm acceleration phase, approaching the time of ball release (Figs 1 to 3); but two of these torques (thorax extension and shoulder flexion) had no discernible local peaks, achieving their maximum values at ball release, although their global maxima would occur after ball release, during the follow-through phase, which was not analyzed in this study.

\section{DISCUSSION}

Fast bowling research has been mainly confined to an analysis of kinematics, a branch of mechanics suitable for describing movement sequences; but to reveal the causal agents of segmental motion, kinetics analysis is needed, on the whole not often performed, the modeling procedure having to derive equations of motion with

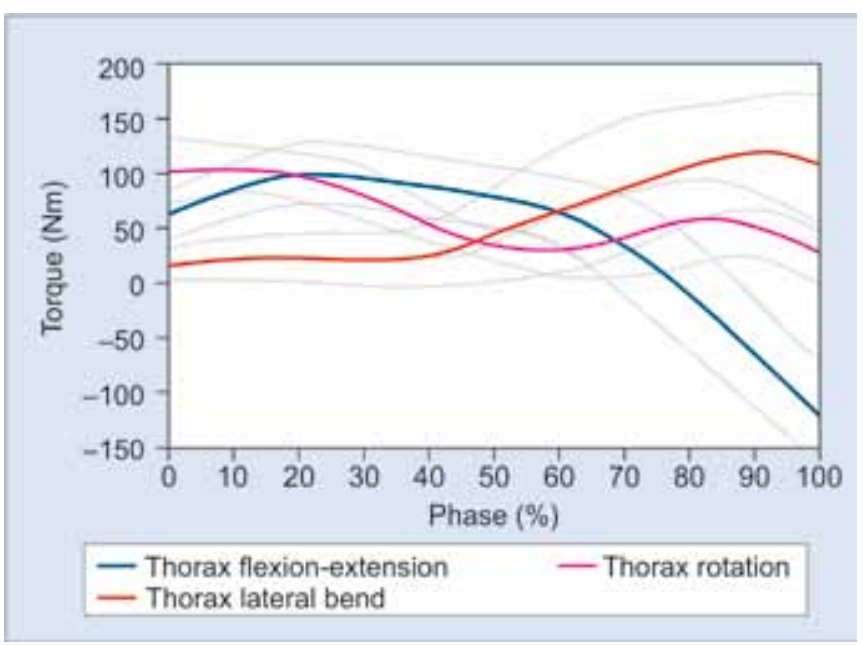

Fig. 2: Ensemble average (mean $\pm \mathrm{SD}$ ) of time-varying joint torques of thorax flexion, thorax rotation and thorax tilt. Flexion, left rotation and left tilt were defined as positive 


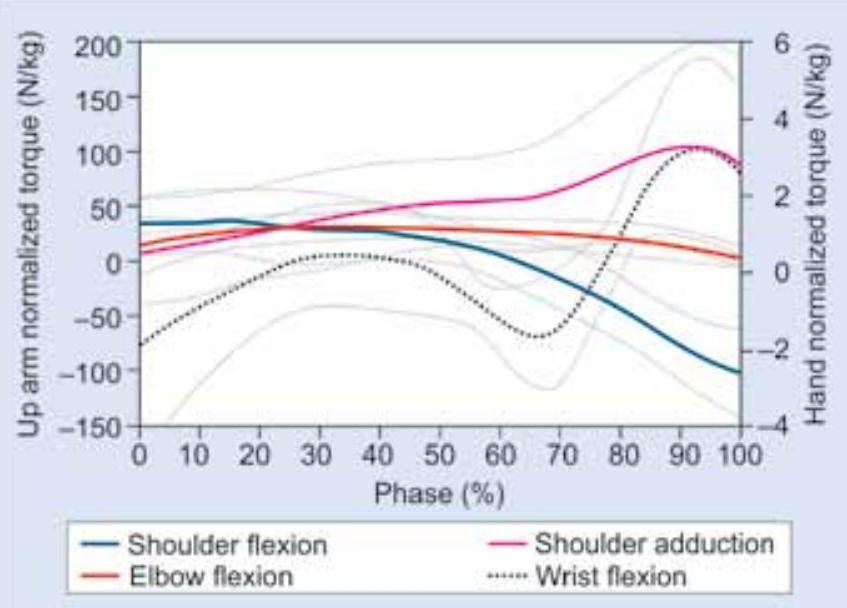

Fig. 3: Ensemble average (mean $\pm S D$ ) of time-varying joint torques of shoulder flexion, shoulder adduction, elbow flexion and wrist flexion. Flexion and adduction were defined as positive

algorithms based on analytic dynamics, a specialized and time-intensive process. To achieve this, a 15 segment rigid body model representing the cricket bowler was created, capable of outputting the torque time-history of fast bowlers tested in a motion analysis laboratory, producing data that potentially reveal the mechanical aspects of effective fast bowling performance.

In terms of torque magnitudes, the largest torques were generally generated by the pelvis segment, a somewhat expected result since it is a large weight-bearing segment comprising a number of large muscle groups; and it is also a segment required to flex, tilt and rotate rapidly in fast bowling - both elements that contribute to the generation of large torques. The peak pelvis torque about each joint coordinate axis was in excess of one times body weight $\times$ height; and considering that this value is multiplied by the number of balls bowled to calculate the workload placed on the lumbar spine in a highly repetitive activity, such as bowling, the peak torque is a factor that determines the cumulative stress and strain placed on the lumbar spine, potentially increasing the risk of lumbar injury, which is one of the most common injuries in fast bowlers. ${ }^{2}$ Hence, bowlers who generate large pelvis torques may be more predisposed to sustaining serious lumbar injury, such as stress fractures of the L4/L5 pars interarticularis. The peak thoracic torques were less than half that of the pelvis torques, but very similar in magnitude to the peak shoulder extension and adduction torques. Considering that the moments of inertia of the arm segments are very much smaller than the moment of inertia of the thorax segment, the effect of shoulder torques on the kinematics of the bowling arm is relatively larger than the effect of thorax torques on the kinematics of thorax, highlighting the importance of shoulder torques in fast bowling-a finding that has potential implications in the development of strength and conditioning programs for fast bowlers. The elbow flexion torque was relatively smaller than the shoulder torques, a result that was consistent with the legal requirements of the bowling action, restricting elbow extension to $15^{\circ}$ during the arm acceleration phase, ${ }^{1}$ essentially making bowling a straight-arm motion. With little or no active motion of elbow involved in bowling, any elbow torques generated would be mostly for the purpose of resisting inertial forces and providing joint stabilization. Furthermore, the elbow flexors are relatively small compared to the larger muscle groups of the pelvis and thorax segments, making it unlikely that the elbow flexors could generate comparably high joint torques.

In terms of quantifying the critical control features that underlie the synthesis of a mechanical system, an analysis of the kinetics sequencing is necessary, particular for determining the complexity of the control processes involved. One thing that is clear from examining the temporal occurrences of peak joint torques is that the kinetic sequencing in fast bowling cannot be reduced to a simple conceptual idea. Classically, the proximal to distal sequence in bowling has been posited as the optimal ordering of segment motions in order to generate high bowling hand velocity. ${ }^{2}$ In terms of the joint torques calculated for the sample of elite bowlers in this study, it is true that the pelvis torques were generally actuated early: the peak pelvis flexion and rotation torques occurring relatively early in the phase (Fig. 1), much earlier than the peak shoulder adduction, shoulder extension and wrist flexion torques-data which is consistent with proximal to distal sequencing. However, there were numerous deviations from this classic sequencing scheme, such as the peak thorax rotation torque occurring first, the peak pelvic flexion torque occurring at ball release, and the lateral bending and flexion-extension peak thorax joint torques occurring late in the arm acceleration phase, closer to the occurrence of the peak shoulder and wrist torques. Whereas if the proximal to distal sequence prevailed, the peak thorax joint torques would have occurred after the peak pelvic joint torques and prior to the peak shoulder and wrist torques.

In summary, the aim of this study was to investigate the fundamental kinetics of important segmental motions during bowling in a sample of elite bowlers. To achieve this purpose, a rigid body dynamics model of the bowler was created in Mathematica's Mechanical Systems Pack, designed with output functions to calculate all the major joint torques during bowling. The model was able to show the time-history of joint torques in fast bowling, enabling a comparative analysis of the pelvis, thorax, shoulder and wrist torques in terms of their relative magnitudes and 
general sequencing. The further development of such kinetics models in cricket bowling will provide further insights into the mechanisms of bowling technique, yielding potential implications for performance enhancement.

\section{REFERENCES}

1. Ferdinands RED, Kersting UG. An evaluation of biomechanical measures of bowling action legality in cricket. Sports Biomech 2007;6(3):315-333.
2. Bartlett RM, Stockhill NP, Elliott BC, Burnett AJ. The biomechanics of fast bowling in men's cricket: a review. J Sport Sci 1986 Oct;14(5):403-424.

3. Grood ES, Suntay WJ. A joint coordinate system for the clinical description of three dimensional motions: application to the knee. J Biomech 1983 May;105(2):136-144.

4. Beretta R. Mathematica Mechanical Systems Pack User's Guide. Champaign, IL: Wolfram Research; 1995.

5. Haug EJ. Computer Aided Kinematics and Dynamics of Mechanical Systems (Vol 1): Basic Methods. Massachusetts: Allyn \& Bacon; 1989. 